\title{
Intelligent Robust Base-Station Research in Harsh Outdoor Wilderness Environments for Wildsense
}

\author{
Junho Ahn ${ }^{1 *}$, Akshay Mysore ${ }^{2}$, Kati Zybko ${ }^{3}$, Caroline Krumm ${ }^{3}$, Dohyeon Lee', \\ Dahyeon Kim ${ }^{1}$, Richard Han ${ }^{2}$, Shivakant Mishra ${ }^{2}$, and Thompson Hobbs ${ }^{3}$ \\ ${ }^{1}$ Korea National University of Transportation, Department of Computer Information Technology \\ 50 Daehak-ro, Chungju-si, Chungbuk 27469, South Korea \\ [e-mail: jhahn@ut.ac.kr, ldh9501@ut.ac.kr,1826059@ut.ac.kr] \\ ${ }^{2}$ University of Colorado, Boulder, Department of Computer Science \\ Boulder, Colorado 80309, USA \\ [e-mail: Akshay.Mysore@colorado.edu,Richard.Han@colorado.edu, Shivakaht.Mishra@colorado.edu] \\ ${ }^{3}$ Colorado State University, Natural Resource Ecology Laboratory, Department of Ecology \\ Fort Collins, CO, USA \\ [e-mail:katizybko@gmail.com,ckrumm@yahoo.com,Tom.Hobbs@colostate.edu] \\ ${ }^{*}$ Corresponding author: Junho Ahn
}

Received December 22, 2020; revised February 5, 2021; accepted February 15, 2021;

published March 31, 2021

\begin{abstract}
Wildlife ecologists and biologists recapture deer to collect tracking data from deer collars or wait for a drop-off of a deer collar construction that is automatically detached and disconnected. The research teams need to manage a base camp with medical trailers, helicopters, and airplanes to capture deer or wait for several months until the deer collar drops off of the deer's neck. We propose an intelligent robust base-station research with a low-cost and time saving method to obtain recording sensor data from their collars to a listener node, and readings are obtained without opening the weatherproof deer collar. We successfully designed the and implemented a robust base station system for automatically collecting data of the collars and listener motes in harsh wilderness environments. Intelligent solutions were also analyzed for improved data collections and pattern predictions with drone-based detection and tracking algorithms.
\end{abstract}

Keywords: Intelligence, Vision Detection, Base Station, Data Collection, Harsh Environments, Robust Base Station, Wilderness Area

This research was supported by Basic Science Research Program through the National Research Foundation of Korea (NRF) funded by the Ministry of Education (No. 2020R1I1A3068274). This work was supported by a grant from the National Science Foundation, IDBR 0754832. 


\section{Introduction}

Monitoring the spread of disease among deer can help biologists and ecologists manage the populations, and they often investigate their movement patterns, locations, and interaction behavior. Wildsense [1] was verified to successfully collect and retrieve the fine-grained data, necessary for monitoring the frequent interactions of wild deer, by retrieving data every $20 \mathrm{~s}$ using a DTN(Delay tolerant networking) $[1,2]$ algorithm installed and operating on the deer collars with the GPS and the RF-radio sensor in a wild harsh environment area. We installed a mechanism on the deer collars that were programmed to automatically detach the collar construction for falling off from the deer's neck and being retrieved. Wildlife ecologists and biologists $[3,4]$ need to wait to collect the collar data for several months or several years until the detachment of the deer collars.

The previous research emphasized the collar node's functionality, importance, and aspects of our software and hardware system design. The collar node collected location and contact information with fault tolerance properties and enables effective operation in harsh real-world environments. However, the previous research lacked investigations of data collection with time saving and low-cost methods for base stations during the deployment period. Ecologists and biologists, sometimes, recapture the same deer to continually keep tracking the health status or collect behavior data. To collect data of collar nodes is required to manage an expensive experiment including a base camp with medical trailers, helicopters, and airplanes to capture deer or to spend much time waiting for several months until the deer collar drops off of the deer's neck.

In this paper, we designed an intelligent robust base-station research and implemented a base station system using a method that is more low-cost and involves less waiting time to obtain recording sensor data from their collars. Readings are intelligently obtained without opening the recaptured deer's collar or during the experiment period when deer, wearing the collar nodes, would be likely to walk by or stand near. We mostly considered the light weight and hardware design of the deer collars since they were easy installed, and the deer continued carrying the collar in the one-month period for harsh outdoor areas. We also focused on the robustness in our system and power consumption of collar and listener nodes for the wildness environments. We tested our listener nodes with each of the six deer collars which recorded sensor data.

This paper makes the following important contributions. First, Wildsense is a low-cost system that is highly robust and fault tolerant in harsh outdoor wilderness environments. Each collar costs less than \$450 US Dollar (USD) and a listener node costs less than \$100 USD with the existing low-performance and low-cost wireless sensors. A wildlife collar for harsh environments costs several thousand dollars in the real world [5]. Location and interaction information is automatically replicated and propagated so that an animal's information can be retrieved in part even if that animal's collar is lost. Second, Wildsense allows intelligently for early data collection in a timely manner. Our previous research lacked investigations of an intelligent base station system with time saving methods during the deployment period. Investigators do not have to wait until the collars fall off and are collected at the end of a deployment to collect some data about interactions.

The paper is organized as follows. Section 2 provides an overview of the background research. Section 3 describes the hardware and software design of the system. Section 4 describes the details of the Wildsense deployment in a wildness area and the data collection process. Section 5 provides a detailed evaluation of the data we collected from our deployment. 
We describe the intelligent research with advanced data collection and analysis methods in section 6 . Finally, section 7 concludes the paper.

\section{Background Research}

Wildsense: We have designed and implemented the Wildsense [1] system to monitor wild deer interaction behavior in rugged wilderness. Wildsense combined the functionalities of both GPS and RF(Radio Frequency)-radio sensors installed in the deer collars. We have demonstrated how our system could function robustly enough to be used in current-day research of wildlife scientists seeking to monitor the spread of disease among deer populations. In contrast to existing commercial systems, our system provides the following three major enhancements in the area of wild animal monitoring. Wildsense was shown to successfully collect and retrieve the fine-grained data, necessary for monitoring the frequent interaction of wild deer, by retrieving the data every $20 \mathrm{~s}$ from the deer collars. We demonstrated how our system successfully monitored the six collared deer's movement patterns and interaction behaviors as they reconnected and interacted with each other: sometimes with the deer from the same herd, and other times with deer from different herds. Our system found that deer in the same herd frequently interact with each other on average approximately $25 \%$ each day within a one-month period. Deer from different herds were found to infrequently interact with each other, only one or two times within a one-month period, for an average of $40 \mathrm{~min}$. Our study also showed that even if a deer collar is not retrievable, our system can still record and measure some of the lost deer's location and interaction data using our DTN algorithm that is run on each deer's collar. Our system was ultimately found to operate robustly in an outdoor rugged wilderness environment for the duration of the research study.

Communication and RSSI: DTN offers a method to relay and collect data in networks that are not always connected. A store and carry forward concept has also been implemented by researches [2, 6-9] where disconnected nodes in the field may be accessed by cattle and the information is relayed back to a sink situated. We adopt a similar DTN-based approach [1] for relaying data because it is particularly well suited to the intermittent contacts between freeranging wild deer. We also investigated LoRa [10] that can exploit its low cost, low power, and long range capabilities in non-urban environments. We mostly considered the light weight and hardware design of the deer collars since they were easy installed and the deer continued carrying the collar in the one-month period as well as robustness in our system and power consumption of collar and listener nodes for the Wildness environments.

The received signal strength indicator (RSSI) is a way for estimating the distance between a transmitter and receiver [11-14]. Jurdak et al. [13] have illustrated the advantage of using RSSI ranging along with GPS to decrease uncertainty of position for outdoor mobile sensor networks. RSSI has also been used to switch on stationary cameras deployed in the wild when monkeys fitted with collars are detected nearby [14].

White-Tailed Deer Ecological Researches: White-tailed deer ecological research [3, 4, 15] used GPS-based systems for tracking their movement patterns and interaction behaviors among a different group and the same group. The deer moves within a small range of several kilometers and frequently interact with each other in the same group. We verified our system's feasibility by comparing the results collected in our system. 


\section{System Design}

We describe how each part of our software and hardware system was designed and built to successfully deploy this base station and collar node experiment, as shown in Fig. 1. The software has to operate without performance reduction or failure over several months. The hardware must be sufficiently weather proof to endure exposure to the elements in the harsh outdoor environments.

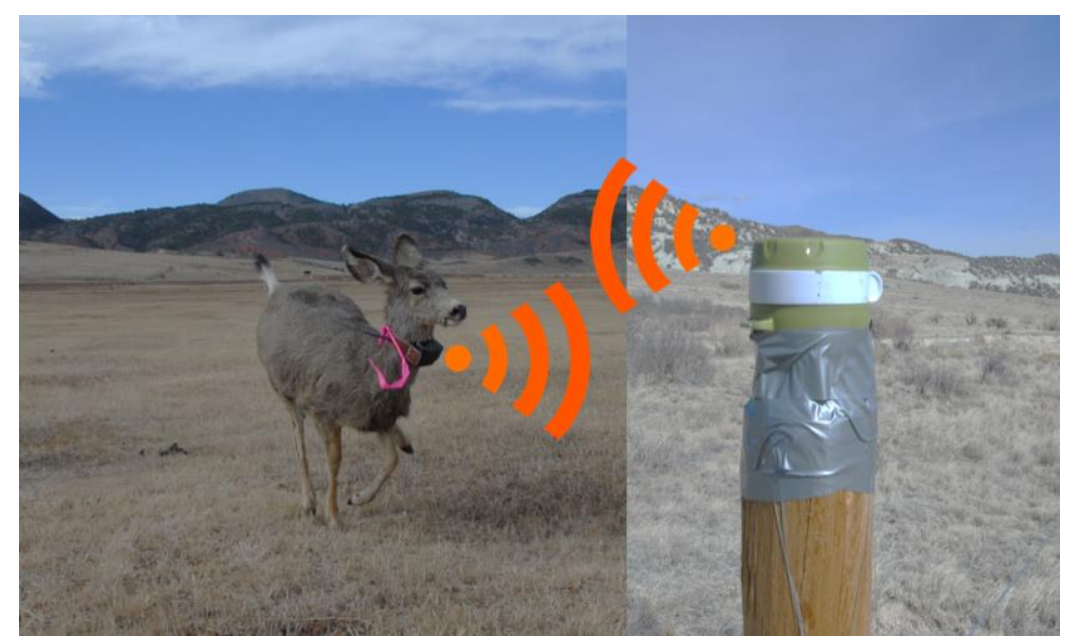

Fig. 1. Base station data collection between a deer collar node and a listener node

\subsection{Data Collection Design}

Tracking and interaction: Our system combines the functionalities of both RF-radio and GPS sensors with minimal-resource and low-cost MICAz motes [16] and is able to operate robustly for a period of up to several months for continual tracking and monitoring of locations and interaction behaviors of wild deer. We utilized time synchronization based on ETA(Elapsed Time on Arrival) [17] to measure time differences in the collar nodes.

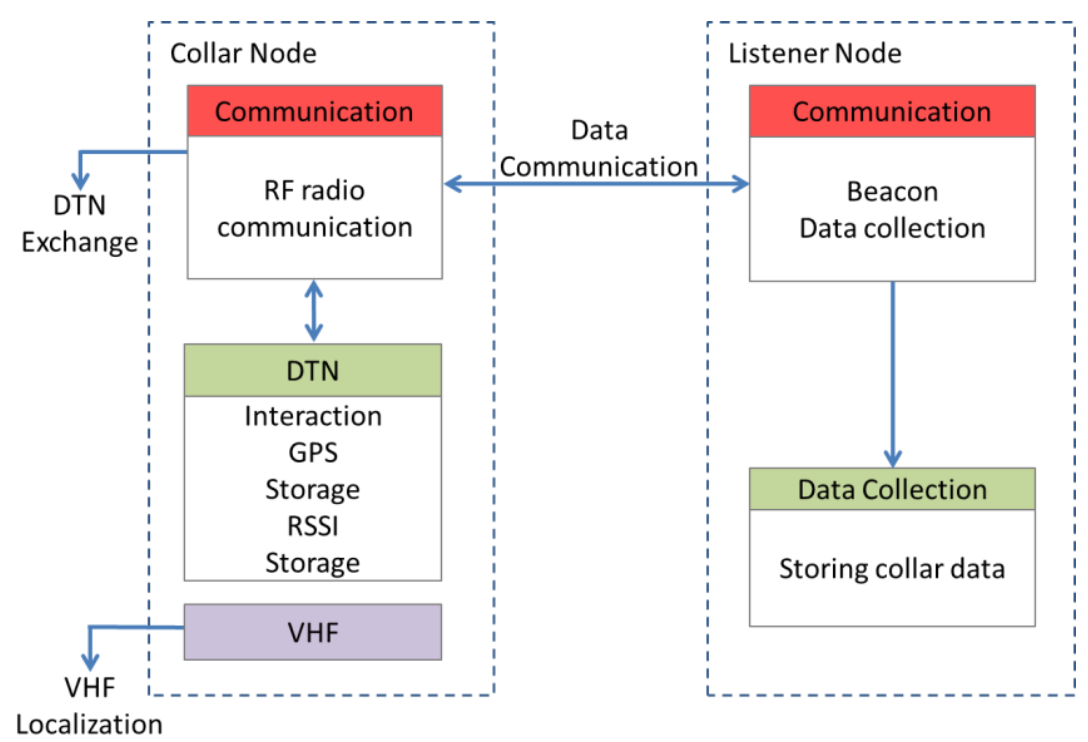

Fig. 2. System architecture between a collar and listener node 
Fig. 2 shows our system's architecture that was designed to monitor deer's locations and interactions with each other through the use of deer collar nodes and a stationary listener node. The collar node collects GPS-based locations and interaction behavior data via RF radio signals within a 30-m range and RSSI, transferred one point data every $6 \mathrm{~s}$. A VHF radio was also installed in each collar node, allowing the collar to be located from its VHF beacon within a maximum $8-\mathrm{km}$ range. The VHF sender board was also programmed to signal to the deer collar to detach on a specific date, approximately 4 weeks after install. A VHF beacon board emits low level and minimal battery power usage signals every second in each deployed deer collar node. VHF cannot be utilized for recording contact information that involves exchanging data packets as it uses analog signals. We recorded the point when we first detected the sound and continued driving until we found the point at which the signal disappeared. With this method, the deer collars in our experiment detached and then fell on the ground as the deer continued to move about. The data recorded on the deer collar nodes is also collected by a stationary listener node whenever deer, wearing the collar, pass by or stand within a 30-m range from the listener node. We describe below each component of our system's software design, and how it operates with the collar and listener nodes.

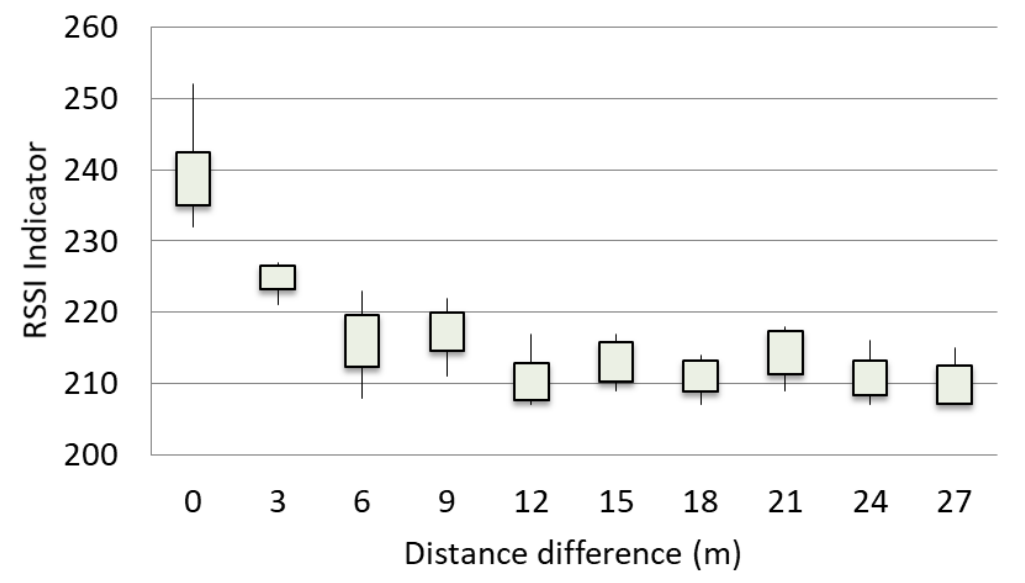

Fig. 3. Relationship between collar received signal strength indicator (RSSI) and distance

We use the radio to measure approximate distance between the two interacting deer collar nodes. Although the indicator cannot measure the specific distance between two nodes, the signal strength can be used to calculate the distance within two levels of ranges: a 10-m and a 30-m range. In the beginning phase of our experiment, in which we captured and then released the deer back into the wild, wearing our collars, we determined that the RF radio signal at 0 $\mathrm{dBm}$ could communicate with neighbor collar signals within a 30-m range, and at a height of $1 \mathrm{~m}$ (the approximate height of each deer's neck). Fig. 3 shows the relationship between the radio strength and the distance measured between the two collars. The Fig. 3 shows a series of boxplots, with the $\mathrm{x}$-axis indicating the distance between nodes plotted against the radio signal frequency on the y-axis.

The rectangular boxes plot the radio signal within + or - one standard deviation based on the average signal for that distance, and the whiskers of the box extend to the minimum and maximum radio signals for that distance. Although the signal strength appears to vary randomly across many of the distances, we discovered a pattern, shown in this graph, in which two different thresholds for approximating the distance between the collars emerged. A 
threshold of a 220 strength signal can be used to determine that two collar nodes are within 10 $\mathrm{m}$ of each other; any signal less than 220 strength meant the collars were within a $30 \mathrm{~m}$ distance range. We also tested the relationship between radio signal and node distance, of our nodes uncovered, and unattached to the deer, and discovered that without the collar covering the nodes, the signal strength was consistently higher at every distance point. When we did an RSSI test, using only an uncovered MICAz mote, the reasonable threshold was 230, but this threshold is reduced when the node is covered by the collar, as shown in Fig. 4.

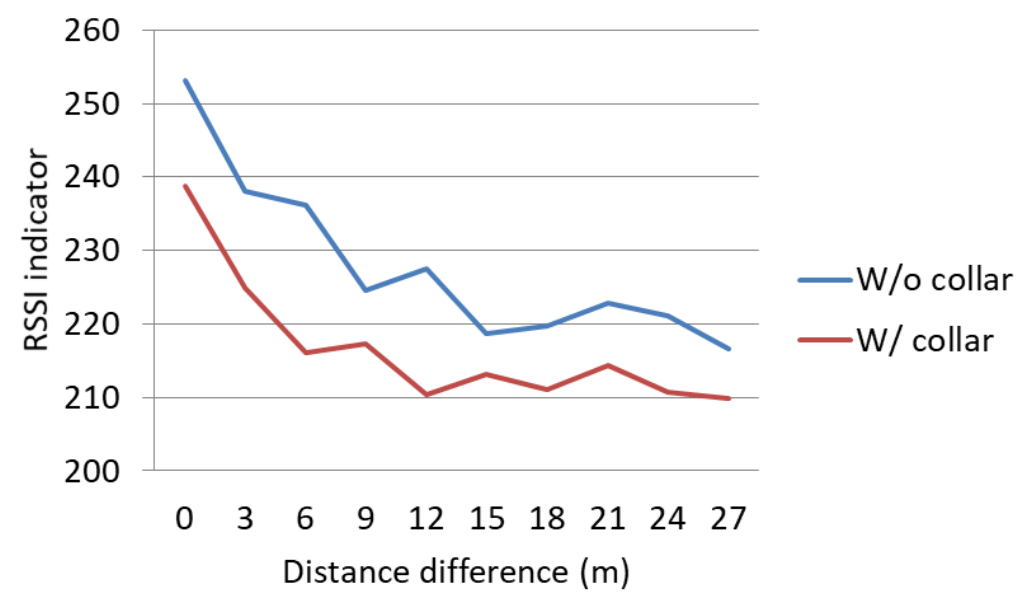

Fig. 4. RSSI performance comparison between with and without the collar

Data collection: Data collection was achieved through two different means. First, we installed stationary listener nodes in different outdoor locations where deer, wearing the collar nodes, would be likely to walk by or stand near. When a collar node detects the presence of a listener mode, that is, when they are within 30-m proximity of each other, the collar node drops into listener mode, whereby it seeks to transfer its current DTN table to the listener as well as dump the contents of its flash $\log$ to the listener. The flash contents are transferred in 64-byte message chunks to the listener.

This method is robust to the deer walking out of the range of listener while the data is being transferred. In this case, only a partial snapshot of the flash contents is retrieved, though this is still useful to provide a timely understanding of how the experiment is proceeding. Both the collar nodes and the listener nodes were programmed to communicate with each other every $6 \mathrm{~s}$ whenever they were within a 30-m proximity of each other. When the deer collar nodes were within range of the listener nodes, the listener nodes could passively collect both the DTN table data and as much of the flash data as could be downloaded from the collar nodes within the 6-s signal period. The collar node data was collected by the listener node, with a passive method-without having to open the deer collars to remove the hardware.

Contact rates at the sub-minute granularity were captured using MICAz RF-radio communication. In the beacon mode, the radios would beacon by default every $20 \mathrm{~s}$, duty cycling to save power by staying up $5 \mathrm{~s}$ and sleeping $15 \mathrm{~s}$ in the sleeping mode. When one deer come into a close range, the collar node begins sending DTN table data with the other node every 6 seconds. If a collar node hears a listener node, it drops out of beaconing mode and into Listener mode. When a collar comes to a listener node into a close range, the listener node drops into Listener mode and begins receiving DTN table data every 6 seconds. These different states are outlined in Fig. 5. 


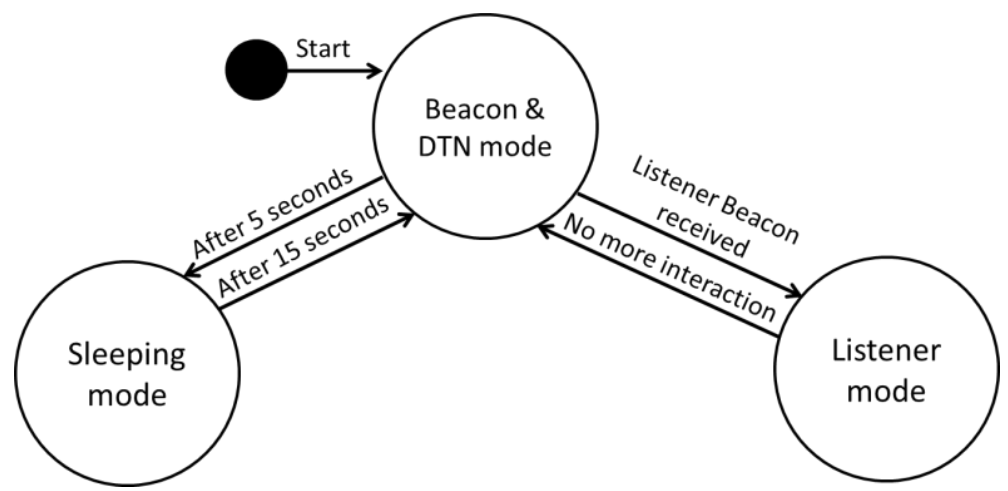

Fig. 5. State diagram of listener node communication

\subsection{Hardware Design}

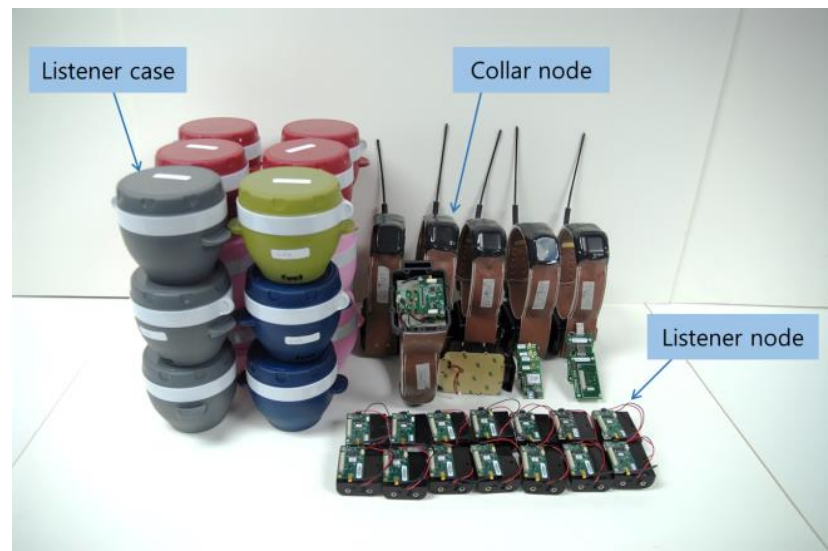

Fig. 6. Listener nodes and collar nodes

We deployed six nodes on deer collars and 14 stationary listener nodes in various locations in the outdoor prairie wilderness area as shown in Fig. 6. The 14 stationary listener nodes each included a MICAz mote, and were assembled within a waterproof plastic box. In the section below we describe the features of the equipment we used in the assembly of the listener nodes.

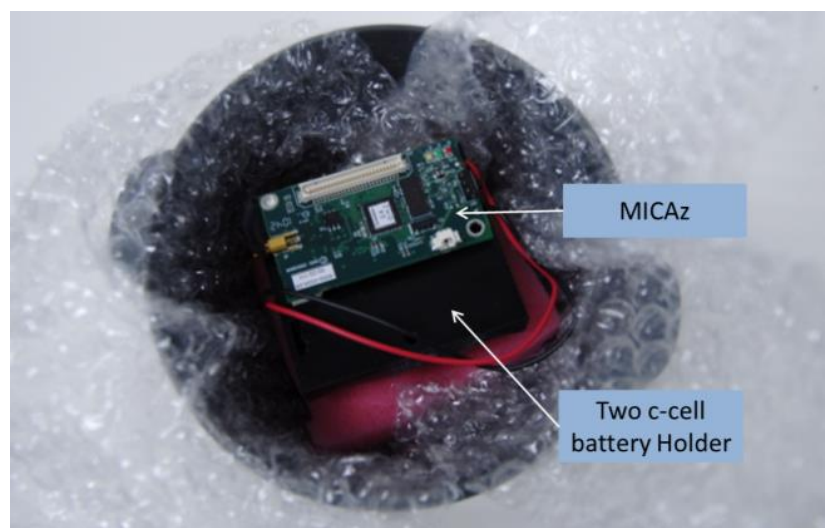

Fig. 7. Listener node with a case 
The case of the listener node was not needed to be as robustly constructed as the collar sensor node since it was installed in a stationary location, as shown in Fig. 7. We designed it to be waterproof, however, to prevent water from snow seeping in to the electronics. In the listener case we placed a spongy cushion, on which we installed the MICAz mote, and then covered the mote with bubble wrap. We connected the MICAz mote to two $1.6 \mathrm{~V}$ c-cell batteries since the listeners were not required to be deployed for a long time, and covered it with bubble wrap and sponge to prevent it from dislodging due to shock. The covered listener nodes were deployed in the outdoor prairie area for a one-week period, with no operating issues.

\section{Deployment}

Our deployment of base station and collar nodes on wild deer consisted of the following steps: collar node deployment, listener node deployment, collar node collection, and listener node collection in the wilderness area.

\subsection{Collar Node Deployment}

Our deployment of collar nodes on wild deer consisted of base camp setup, a small search airplane, deer capture by helicopters, installation of collars on deer, and the release of deer. We built a base camp in the wilderness area and conducted an airplane search for wild deer that were then caught using two helicopters. The 20 members of the research team consisted of ecologists, biologists, a ranger to manage the park, and engineers. The team arrived at the wilderness area at approximately 7 a.m. on the first day of the experiment, and searched for a reasonable place to build the base camp. After choosing a suitable area for the base camp, the team brought in a deer searching airplane, two helicopters, fueling trucks, and a medical truck.

In order to capture the free-range deer, we first needed to locate the deer, but the wilderness area was quite large and not easy to quickly search. Although the helicopter could have been used to both locate the deer and capture them, it requires much more fuel than an airplane and is limited in the time it can be airborne between fueling stops. It was not feasible or economical to use the helicopters for searching for the deer, thus we utilized a small search airplane to locate free-range deer in the large area surrounding the base camp. The search plane flew over the wilderness area for long periods of time, and when it finally located any deer, the researcher in the plane notified the team at base camp of the deer's location.

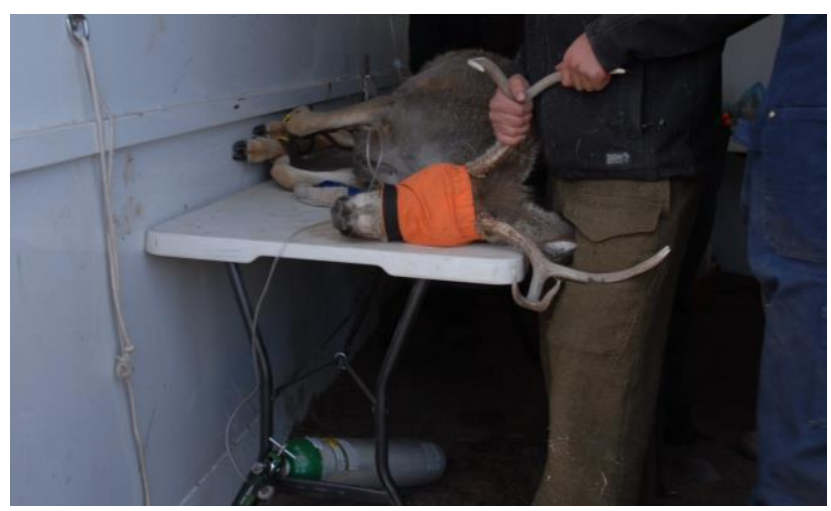

Fig. 8. Deer being carried into the medical trailer 
Up to two helicopters were used to capture the deer after the search plane notified the pilots of the locations of one or multiple deer. The researcher, from the helicopter, gave the deer the drug that quickly made the deer lethargic and motionless. The researcher then bound the deer's legs and blindfolded the deer. Biologists gave it a general checkup and then the collar was installed on the neck of the captured deer in Fig. 8. They released the deer back into the wilderness area.

\subsection{Listener Node Deployment}

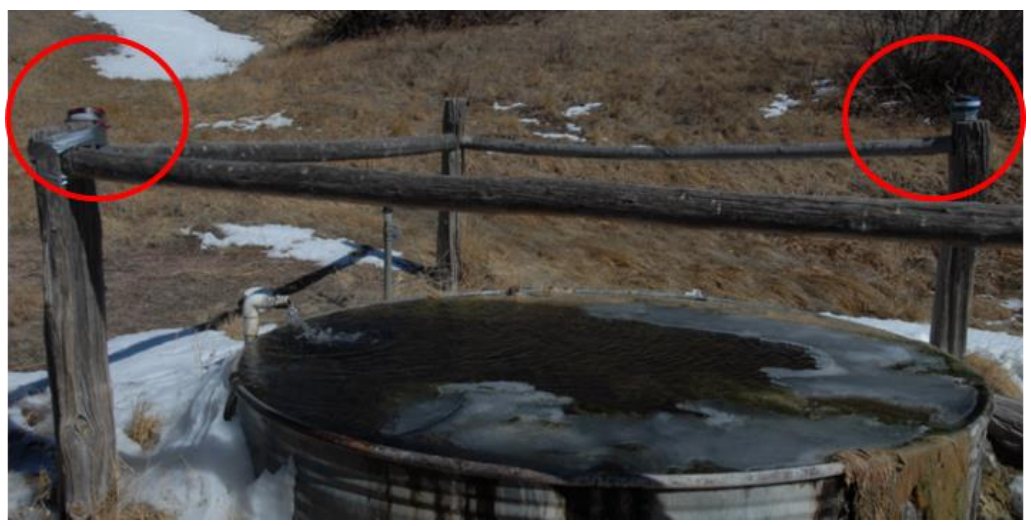

Fig. 9. Listener node deployment near a water tank

For our listener node deployment, we asked ecologists, who were familiar with the area and deer's habits, to advise us on the best locations in which to install our listener nodes. We utilized fourteen listener nodes to cover a wider area than one node's communication area and improve the probability for data collection from deer collar nodes on the locations. We deployed the 14 listener nodes in areas where the ecologists expected deer-including those wearing our collars - to congregate or visit. We deployed our listener nodes to collect data from the six deer collars in four places. We deployed our listener nodes near a water tank, as shown in Fig. 9, and on a tree, as shown in Fig. 10. We also installed the listener nodes on two different location's fences, as shown in Fig. 11, and on the medical trailer truck, as shown in Fig. 12. There were several water tanks in the area, many different fences and many trees to choose from, as would be expected.

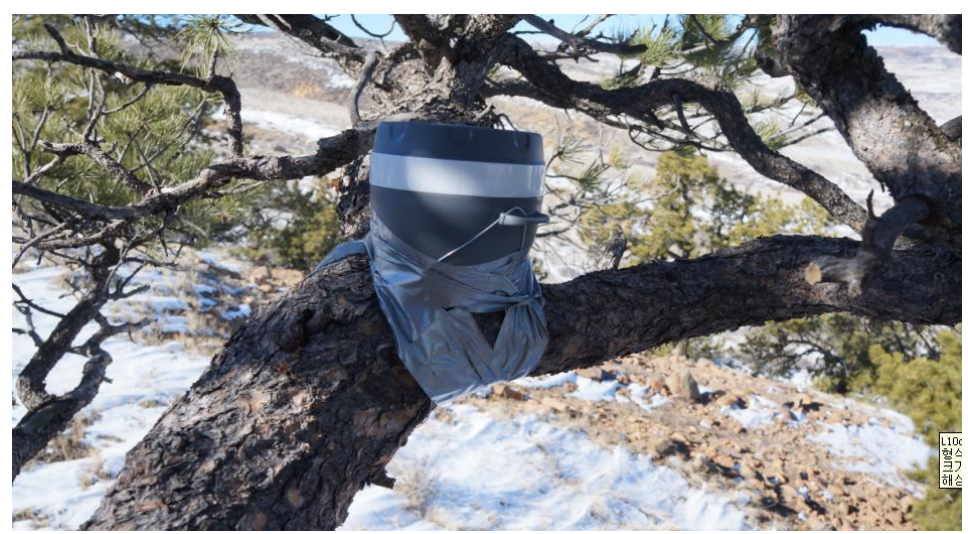

Fig. 10. Listener node deployment on a tree 


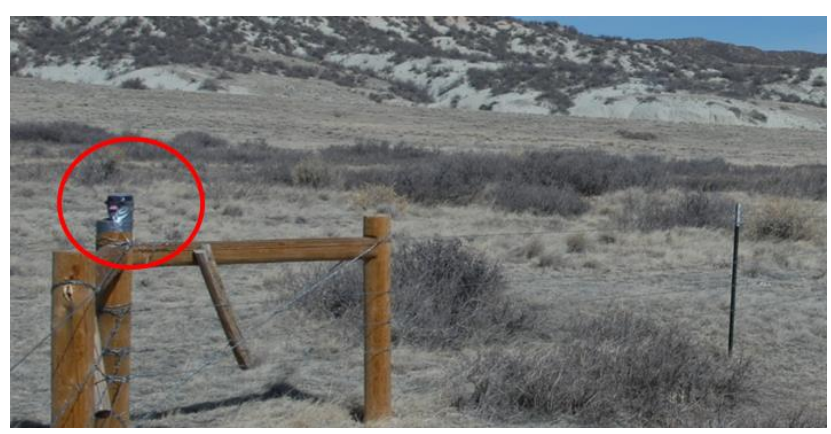

Fig. 11. Listener node deployment on fences

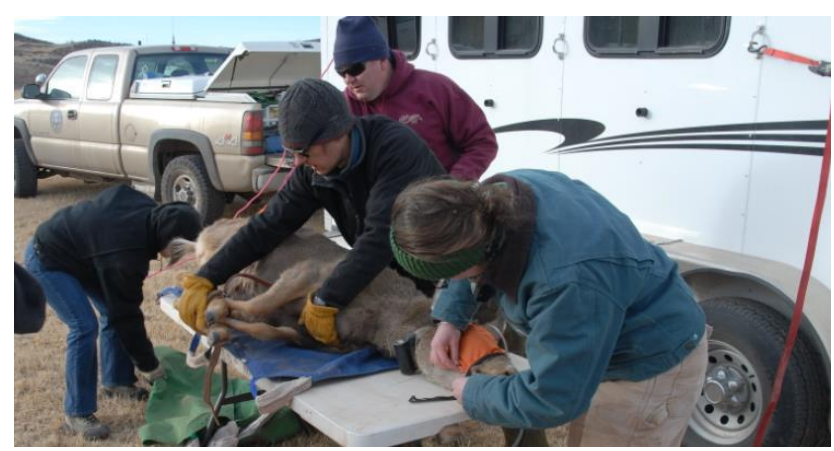

Fig. 12. Listener node deployment on medical trailer trunk

Thus, in order to find the best position to install our listener nodes, we used a VHF searching method. While walking across the land in search of a dropped-of collar whose signal we had detected, we hand-carried the receiver and a small antenna attached to it, as shown in Fig. 13. The deer collars include a VHF signal beacon and we could approximately locate them using a VHF receiver. We used a VHF receiver, which can detect the sender within about $8 \mathrm{~km}$, and could approximate the location of the deer with our collars. Using the VHF receiver's antenna, we could approximate the location of the deer by the intensity of the receiver's beeping signal the closer it got to the deer's collars. We installed a total of 14 listener nodes near the locations where we received the strongest VHF signal: two nodes on the water tank; one node in the tree, five nodes each on two different fences, and; one node on the truck. At the end of the week in which we tested these listener nodes, we retrieved all of them from their various locations.

Normally, wildlife ecologists and biologists capture and recapture deer to track their health and disease status as described in the collar node deployment section. The listener node installed on the truck was used to test a simulated recapturing method for future use for wildlife researchers. We tested our listener node-recapturing method with each of the six deer we had captured and collared. Although this was only a simulation in that our experiment did not provide the time to capture and recapture deer, the procedure we employed would be the same with recaptured deer. Deer with collars could be recaptured to collect newly obtained health status data and recorded sensor data from their collars in a 20-min period, and readings could be obtained without opening the recaptured deer's collar. Once the deer's data was collected from its collar by the listener node, it could be rereleased into the wild with its collar still deployed. Fig. 12, shows the truck with the listener node adjacent to the medical personnel examining the deer. 


\subsection{Collar Collection}

We began collecting the detached deer collars in the Soapstone prairie land, after three weeks into the project. The deer collars had all been deployed with a VHF beacon attached to them. We used a VHF receiver that picks up the signal from the senders to locate the collars. Each individual collar had been programmed with a unique VHF frequency. Fig. 13 shows the equipment we used to locate the deer collars: a VHF receiver; a GPS device, and; a pair of binoculars. Two team members covered the deployment area by driving back and forth across the land on dirt roads, attempting to pick up the signals from the six different collars. The truck had an antenna attached to the roof, which could pick up the deer collar signals more easily. As we drove, we were able to search for the six different collars by continually cycling through the frequency numbers on the receiver to the six programmed collar-sender frequencies until a signal was identified. The VHF signal covers a wide range of up to $8 \mathrm{~km}$ in radius. The receiver could detect whether the VHF beacon's signal was coming from a collar still attached to a deer's neck or from a collar that had already dropped off of a deer's neck. If the receiver registered one beep per second it indicated to us that the collar was still attached to the deer's neck. When the beep signal was detected at a rate of twice a second, this indicated that the collar had dropped off of the deer's neck, because there was no longer a body barrier or any movement of the deer interfering with the signal. Once we could identify a signal coming from one deer's detached collar, we recorded the point when we first detected the sound and continued driving until we found the point at which the signal disappeared. Based on these two points, we mapped out a circular area on which the collar could be found. We then drove back to the center of the circle and got off the truck to walk back and forth across the area until we located the collar. We spent approximately 80 percent of our search time traversing across the prairie land on foot in order to locate the detached collars.

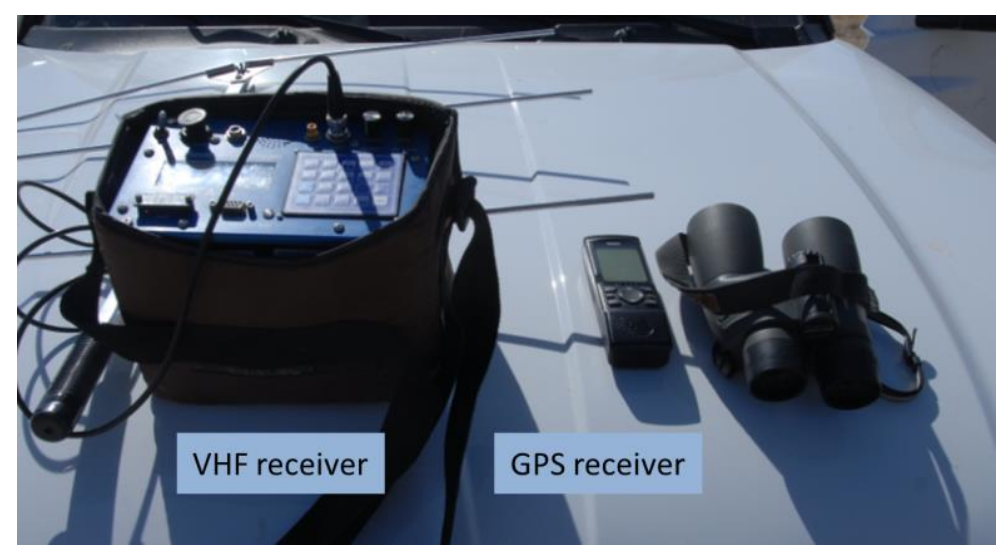

Fig. 13. Collar locating equipment

While walking across the land in search of a dropped-of collar whose signal we had detected, we hand-carried the receiver and a small antenna attached to it. We continued to hold the antenna and point it in the direction of which we were getting the loudest signal as we searched the area. Sometimes the VHF signal would suddenly get weaker as we walked in the direction of the original because of land barriers, such as hills and valleys. For this reason we had to continually check for the strongest signal and keep traversing the area with the antenna held high in front of us as we searched for the collar. 


\section{Data Analysis}

We have analyzed listener and collar data retrieved from nodes. Our evaluation focused on analyzing node robustness without performance reduction or failure, and interaction data over time.

Listener Node: Initially in the experiment we were advised by the ecologists of where to place our stationary listener nodes, based on their assumed knowledge of where they expected the deer to most frequently visit. We placed 13 listener nodes at the places identified by the ecologists: fences on the prairie land, a water tank and in trees out on the prairie. Unfortunately none of the six deer passed by these listener node locations, and so we were unable to collect any data from these nodes. However, we placed the fourteenth listener node on the base camp truck and simulated while walking by or standing near. When a deer wearing a collar node is brought to base camp, the GPS tracking and interaction data collected to date on the deer's collar can be read passively by the listener node without having to open the collar and dump the data.

During the simulation method, we found that we could collect 200 data points (a maximum of eight days of GPS information, one data point collection every $6 \mathrm{~s}$ ) within 20 min. Each collar node records GPS location sensor data directly on the flash every hour, as well as interaction data when two deer come into close range. The GPS location data collected from the recaptured deer could inform the biologists and ecologists of where the deer had visited recently so that we can then place additional listener nodes in those places.

We evaluated robustness among the weather-proof base stations and collar nodes in various different types of terrain or weather in rugged, harsh outdoor environments for one month. Our system functioned and operated flawlessly throughout the 30-day experiment period. We also collected data points from deer collar node through a one-month deployed listener node with RF-radio communication after finishing the experiment without opening weather-proof deer collar. The listener node received all the data points ( 2484 points with interaction data) from the collected collar node without any communication loss. We evaluated the hardware robustness of both listener and collar nodes in outdoor wildness environments.

Furthermore, the method can be extremely valuable for wildlife biologists and ecologists when monitoring deer behavior for extended periods of time. For instance, perhaps deer collars could be designed to not detach and fall from the deer's necks so that a deer could be tracked for months at a time. Also, once more accurate locations are found in which to deploy the listener nodes, the deer could remain in the wild and the data could intermittently be read by the stationary listener nodes.

Interaction Distance: We analyzed the interaction distance between two deer who were captured at the same time from the same herd. We have also analyzed the possible distance between deer during their interaction using a combination of RF-radio communication data and radio strength value (RSSI). Whenever the deer shared their DTN data, the nodes recorded that the deer were within $30 \mathrm{~m}$ of each other. In addition, they also recorded the RSSI value of the signal and stored that data on the flash. If the radio strength value was higher than a 220 threshold strength, the two deer were known to be interacting with each other within a 10-m range, as shown in Fig. 3. When the RSSI value was measured to be less than 220 strength, we could only know that the deer were somewhere within the 30-m RF-radio signal range of each other. We calculated the proportion of time the deer's distance proximity was within a known 10-m range or a 30-m range, as shown in Table 1. We compared these two percentages for deer from the same herd with deer who were from different groups, and found that for deer within a 30-m range of each other, the deer from the same herd were slightly more frequently found to be within a $10-\mathrm{m}$ range of each other than the deer from different groups (14\%, from 
same herd; $12 \%$ from different groups). In addition to the geographic locations of deer, we are also able to track the movement of individual deer over time.

Table 1. Interaction distance proximity: deer from same herd vs. different herd

\begin{tabular}{|c|c|c|}
\hline RSSI & Within 10 m & Within 30 m \\
\hline \hline Same herd (\%) & $14.3 \%$ & $85.7 \%$ \\
\hline Different herd (\%) & $12.8 \%$ & $87.2 \%$ \\
\hline
\end{tabular}

Scientific Inquiry: Ecologists and biologists on our team were very impressed with the results of our experiment in tracking and recording deer's location, movement and interaction. As demonstrated in this section, Wildsense allows scientists to study social behavior of animals in their natural habitat with minimal intervention. While location tracking was feasible from earlier telemetry systems, the ability to record interactions at much finer time granularity, the ability to gather data about lost animals, the potential to analyze the data before an experiment is completed, as well as robustness and low cost are some of the unique characteristics of Wildsense. In particular, research related to monitoring the spread of contagious diseases among deer herds that some of the scientists in our team are involved in will benefit significantly from Wildsense.

\section{Intelligence Solutions for Collections and Data Analysis}

\subsection{Drone-based Vision Detections}

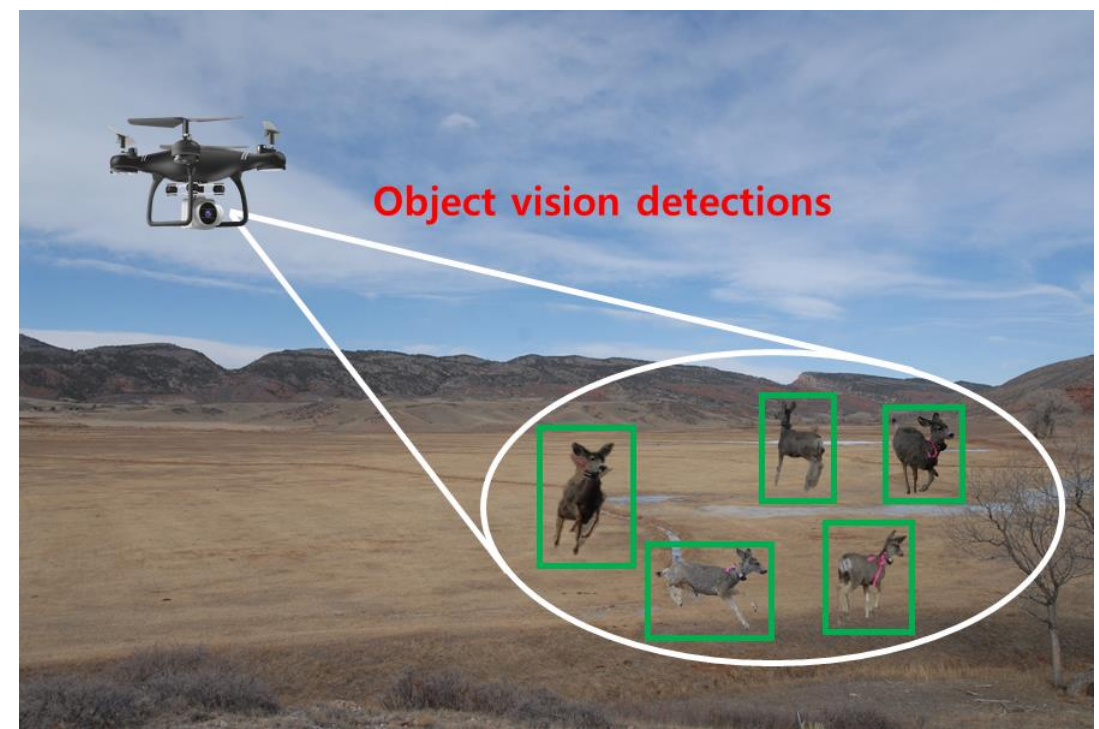

Fig. 14. Intelligent drone-based object vision detections for deer group identifications

Existing methods for detecting the wild deer that wore sensors within a range of $8 \mathrm{~km}$ had limitations, such as a passive approach and a long detection time. To solve these problems, this study proposed a drone-based detection method for the rapid and automatic tracking of the deer as shown in Fig. 14. A VHF receiver is used to identify, approach a deer within a range of $8 \mathrm{~km}$ in a wild environment, and obtain a signal from the sensor worn by it. If the collar node is confirmed within a range of $8 \mathrm{~km}$ based on the VHF receiver, the location of the 
node should be quickly identified for approach. Although existing methods confirm the location of deer within a range of $8 \mathrm{~km}$ based on VHF receivers, they detect the location of deer by a passive approach depending on the signals received from VHF receivers and human eyesight. For this reason, these methods are considerably time consuming. On the other hand, drones can be employed as better alternatives [18-22] to human eyesight to automatically identify and detect the location of deer in the air in a shorter time.

VisDrone research [21, 22] compared the performance of object detection algorithms learned by VisDrone-Datasets, which were Vision datasets obtained by drones under different heights and location conditions. These object detection datasets applied manual annotations to ten categories, including people, bicycles, and automobiles. They examined the performance of object detection algorithms which learnt the VisDrone-DET2018 dataset [21] and the VisDrone-DET2019 dataset [22] and found that HAL-Retina-Net, DP-Net, DPNet-ensembel, RRNet, and ACM-OD were among the $\sim 70$ algorithms that exhibited higher performance than the other algorithms.

HAL-Retina-Net is an object detection algorithm based on Retina-Net [24], which combines ResNet with FPN [23]. It contains the pyramid structure of Retina-Net adjusted to facilitate the detection of objects based on low-resolution image data obtained from drones. It includes Soft-NMS [25] and bounding box voting in the post-processing step to increase the detection accuracy. In addition, it applies a hybrid attention mechanism based on the squeeze and excitation module [26] and downsample-upsample [27].

DP-Net and DP-Net-ensemble are object detection algorithms that employ FPN to learn RCNN-based algorithms, such as faster R-CNN [28] and Cascade R-CNN [29]. Both object detection algorithms can provide a feature quantification function based on RoIAlign [30] that replaces RoIPooling and detects multiple-scale objects in different sizes. They apply SoftNMS and NMS to the predicted box selection of the detected objects.

ACM-OD is an object detection algorithm based on faster R-CNN applying FPN. Initially, this algorithm localizes small objects by learning in the chip area [31] where ground truth samples are concentrated. Subsequently, it performs patch-level augmentation to reduce class imbalance and high false positive (FP) ratio and repeats the entire process to increase the performance of object detection. Methods [32] to increase the accuracy of this algorithm by changing the scales and aspect ratios and applying box voting to a post-processing step instead of the existing learning method have also been developed and applied to this algorithm.

RR-Net is a Center-Net [33] object detection algorithm that uses CNN to predict the center points and sizes of class objects. It creates a coarse bounding box based on the predicted center points and sizes and transfers it to a re-regression (RR) module to calculate the bias for adjusting the location of the box. This process generates the final bounding box based on SoftNMS.

\subsection{Drone-based Tracking for Data Collections}

This study proposed a mobile base-station system that would solve the problem of the existing fixed method of collecting data accumulated in a collar node worn on a deer during the experiment. Researchers should continuously maintain the location of the listener node within a range of tens of meters compared to the location of the deer to obtain data accumulated in collar nodes worn by them. The proposed mobile base-station system adopted an automatic tracking system of drones as shown in Fig. 15, instead of an existing fixed method requiring the placement of listener nodes in a certain location. When the data is exchanged between the collar node worn on a deer and the listener node, both nodes should be located in an available transmit and within range until data collection is complete. The existing method requires a 
fixed positioning of the listener node in an area where a deer wearing a collar node primarily lives. For this reason, problems arise when the deer wearing the collar node exits the transmitting and receiving range. The GPS-based automatic tracking system included in drones can be used to solve this problem where a specific deer wearing the collar node can be continuously tracked within the transmitting and receiving range.

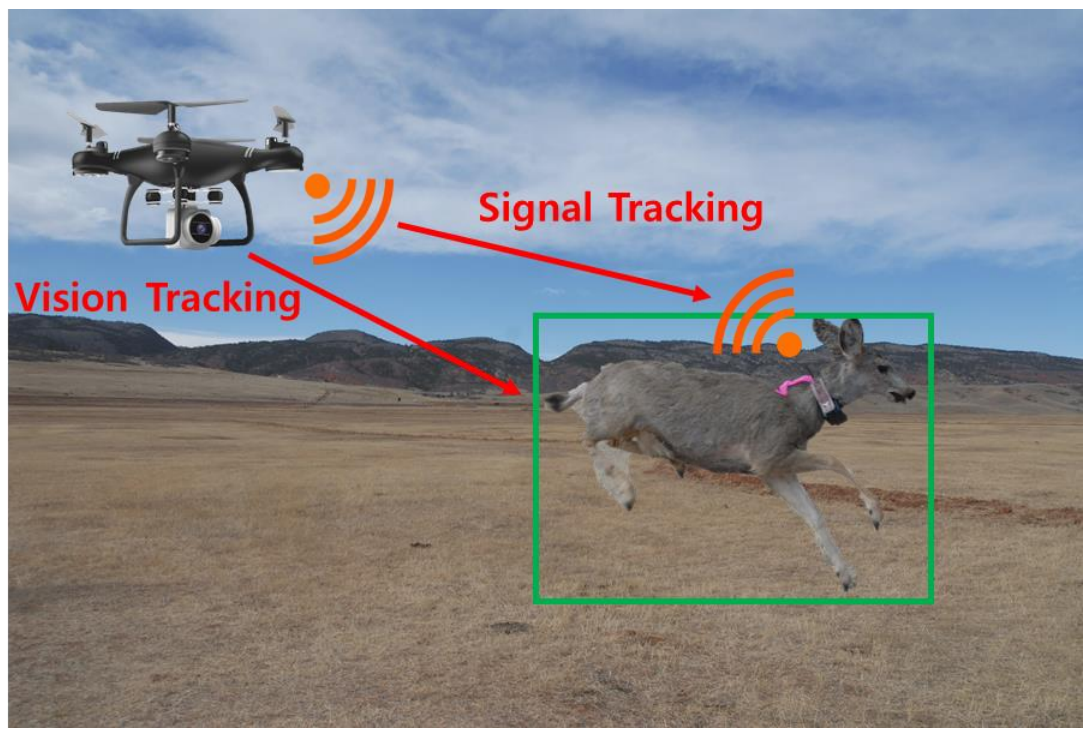

Fig. 15. Intelligent drone-based vision and signal tracking for data collections of collared deer

Table 2. Comparison of the performance of drones equipped with the follow-me function

\begin{tabular}{|c|c|c|c|c|}
\hline Component & Skydio 2 & DJI Mavic Pro & Typhoon H & Walkera Scout X4 \\
\hline $\begin{array}{c}\text { Recognition } \\
\text { Method }\end{array}$ & $\begin{array}{c}\text { Vistion Sensor } \\
\text { + GPS }\end{array}$ & $\begin{array}{c}\text { Vistion Sensor } \\
\text { + GPS }\end{array}$ & GPS & GPS \\
\hline $\begin{array}{c}\text { Vision } \\
\text { Tracking }\end{array}$ & O & O & X & X \\
\hline $\begin{array}{c}\text { Signal } \\
\text { Tracking }\end{array}$ & O & O & O & O \\
\hline Flight Time & $23 \mathrm{~min}$ & $27 \mathrm{~min}$ & $25 \mathrm{~min}$ & $25 \mathrm{~min}$ \\
\hline $\begin{array}{c}\text { Max Flight } \\
\text { Speed }\end{array}$ & $58 \mathrm{~km} / \mathrm{h}$ & $50 \mathrm{~km} / \mathrm{h}$ & $70 \mathrm{~km} / \mathrm{h}$ & $60 \mathrm{~km} / \mathrm{h}$ \\
\hline Camera Pixel & $45 \mathrm{MP}$ & $12.7 \mathrm{MP}$ & $12.4 \mathrm{MP}$ & $13 \mathrm{MP}$ \\
\hline $\begin{array}{c}\text { Wind } \\
\text { Resistance }\end{array}$ & $40 \mathrm{~km} / \mathrm{h}$ & $29-38 \mathrm{~km} / \mathrm{h}$ & $32-67 \mathrm{~km} / \mathrm{h}$ & $30 \mathrm{~km} / \mathrm{h}$ \\
\hline Flight Height & $4.5 \mathrm{~km}$ & $5 \mathrm{~km}$ & $0.122 \mathrm{~km}$ & $0.3 \mathrm{~km}$ \\
\hline
\end{tabular}

Recently developed drones generally include cameras and a follow-me function that focuses on a photographic function. The follow-me function is as an automatic tracking system that detects a particular object through GPS-based location identification or the combination of the above-mentioned method and vision recognition. Because this function can be used to identify the location of a transmitter equipped with the GPS, it can be used to identify the location of a deer by detecting the GPS included in the collar node worn on the deer to 
automatically track the deer on the move, and collect data from the collar node. Table 2 compares the performance of well-known drones equipped with the follow-me function.

Drones such as Typoon H [34] and Walkera Scout X4 [35] can identify and track the location of the transmitter using the GPS-based follow-me function. Recently released drones include an enhanced version of the follow-me function in which vision recognition is additionally applied to detect a moving object and ultimately increase the performance of tracking objects. Representative drones applying the latest follow-me function, such as Skydio 2 [36] and DJI Mavic Pro [37], can detect moving objects in videos recorded by cameras included in them and automatically track certain objects selected by remote controllers. In this regard, the tracking performance of drones is expected to be enhanced by the application of vision recognition to the existing GPS-based deer tracking method.

\subsection{Intelligent Analysis of Behavior Patterns}

Table 3. AI behavior pattern analysis algorithms

\begin{tabular}{|c|c|c|c|c|}
\hline Algorithm & Data Collection & Kind & Recognition Result & Based \\
\hline \hline PRNN & $\begin{array}{c}\text { GPS + } \\
\text { Environmental Data } \\
\text { (terrain, weather) }\end{array}$ & Long-billed & $\begin{array}{c}\text { Repeated } \\
\text { movement pattern }\end{array}$ & $\begin{array}{c}\text { RNN, CNN, } \\
\text { LSTM, } \\
\text { Random Forest }\end{array}$ \\
\hline H2O-DL & GPS + TDR & $\begin{array}{c}\text { Shags, } \\
\text { Guillemots, } \\
\text { Razorbills }\end{array}$ & $\begin{array}{c}\text { Repeated diving } \\
\text { pattern for hunting }\end{array}$ & $\begin{array}{c}\text { H2O, Artificial } \\
\text { Neural Network }\end{array}$ \\
\hline moveHMM & GPS & $\begin{array}{c}\text { Wild } \\
\text { Haggis }\end{array}$ & $\begin{array}{c}\text { Repeated } \\
\text { movement pattern }\end{array}$ & HMM \\
\hline TECA & GPS & Traveler & $\begin{array}{c}\text { Domain-based } \\
\text { movement pattern }\end{array}$ & Semi-Supervised \\
\hline $\begin{array}{c}\text { Markov } \\
\text { chain+MNL }\end{array}$ & GPS & $\begin{array}{c}\text { Domain-based } \\
\text { movement pattern }\end{array}$ & HultiNomial logit \\
\hline
\end{tabular}

The state-of-the-art AI algorithms for animal pattern analysis can efficiently and accurately analyze and predict behavior patterns using data of deer location and interactions collected in wild environments. Various types of pattern recognition AI algorithms [38-44] can be used to analyze animal behavior patterns, and such pattern data can be applied to predict animal behaviors. Therefore, several studies [38,39] on animal behavior pattern analysis have been conducted. Table 3 compares different algorithms [40-44] which can analyze behavior patterns using data of the location and interactions of wild animals and people.

The predictive recurrent neural network (PRNN) [40] is an algorithm applying the adjusted recurrent neural network (RNN) based on deep learning and used to analyze animal behavior patterns and predict their movements. It uses a RandomForest algorithm to interpolate the animal data obtained based on the GPS.Both data of animal behavior patterns and characteristics of the wild environment in which they live are used in the interpolation process. Interpolated data is converted to a density image that represents a trend of animal movements through grouping. The PRNN learns the data transformed into an image using PredRNN++ composing a 5-layer cascade long short-term memory (LSTM) to generate a model and predict animal movements.

H2O-DL [41] is an algorithm that performs learning based solely on GPS data and establishes artificial neural networks using an open source platform called $\mathrm{H} 2 \mathrm{O}$ in R to predict animal movements. This algorithm uses data collected from the GPS, and the size of an input 
layer varies depending on the number of input variables. Four hidden layers are included, and each layer is made up of 20 to 1,000 hidden nodes. This algorithm [41] predicted the location of the diving movements by training this algorithm to learn data from three different types of seaweeds collected from the GPS.

MoveHMM [42] is an algorithm that can analyze and predict data of animal behaviors using hidden Markov models (HMMs) in R. The HMM is one of the Markov models and includes an observation state and a hidden state. The observation state is used to estimate the hidden state. A time sequence is crucial to the analysis and prediction of animal behaviors where these behaviors have a continuous relationship. In this sense, this algorithm learns data in accordance with a time sequence instead of a random approach to generate a model.

Algorithms for analyzing human behavior patterns instead of wild animals based on the location of smartphones and activity sensors can carry out precise and accurate predictions through deep learning. These algorithms can also be applied to the analysis of animal pattern.

A semi-supervised convolutional autoencode (SECA) [43] is an algorithm that predicts the following location by learning both a small amount of GPS data including labels and a large amount of GPS data not including labels through convolutional neural networks (CNNs) based on deep learning. This algorithm devices GPS data into segment sets and transforms each GPS segment into a 4-channel tensor. This algorithm learns the transformed GPS data, including labels, using a CNN classifier. In the process of learning GPS data, not including labels, it exchanges weight data with a convolutional-deconvolutional autoencoder (Conv-AE). When a GPS value is entered in the trained SECA model, the following GPS location is predicted.

An algorithm [44] combining both a Markov chain and multinomial logit (MNL) predicts the next destination a traveler is expected to visit based on the GPS data only. This algorithm applies both the MNL algorithm and the Markov chain algorithm, which serves as the basis for the moveHMM algorithm mentioned above. Markov chain is one of the Markov models and assumes that time and status are independent and that a certain status depends only on a directly prior status. This algorithm [44] applies the MNL model, one of the habit-based models, as well because traveler's travelling habits are reflected when the traveler selects a destination.

\section{Future Work}

Behavioral pattern analysis and prediction methods can be applied to predict the spread of epidemics based on the continuous collection of data of wild animal movements, as shown in the previous study [45]. The advice to the public announced by the World Health Organization (WHO) [46] states that people and animal movements have been restricted and controlled as a result of the global spread of coronavirus disease 2019 (COVID-19) in 2020, which implies the severity of epidemics spread by movements. Applying behavioral pattern analysis and prediction methods in combination with epidemic spread prediction algorithms will help managers and civil servants in the public health department to prevent the spread of epidemics. With regard to studies predicting the spread of epidemics such as COVID-19 [47, 48] predicted the spread of infectious diseases affected by environmental factors, such as climate according to latitude, weather, and air pollution. Studies on predicting the spread of infectious diseases affected by movements are as follows: Disease propagation estimation studies $[49,50]$ predicted the spread of Influenza A virus subtype (H1N1) based on movements in 2009. Research [51] predicted the spread of Ebola virus disease (EVD) which occurred severely between 2014 and 2016 using movement models. Research [52] predicted the spread and peak of COVID-19 in 2020 by employing a SEIR model that applied deep learning based on the 
LSTM. Other propagation researches $[53,54]$ also predicted the spread of COVID-19 by using a susceptible-infected-removed (SIR) model, a fundamental model for predicting the spread of epidemics.

Additionally, the drone research for collecting collar data in real time is limited to the battery life during the flight time. Most commercial drones can have a flight only about half hour and it needed to charge the batteries within $60-90$ minutes [55]. We expect that the limitation of drones is improved to effectively collect data from deer collar.

\section{Conclusion}

Our system ultimately operates with very low-cost and minimal-resource motes that were shown to robustly function in harsh outdoor environments for an extended period of time. Listener nodes were shown to successfully collect the DTN node data from the deer collars during the tracking experiment period. Existing methods for approaching and detecting wild deer within a range of $8 \mathrm{~km}$ based on VHF receivers have limitations, such as a long detection time and passive detection. To circumvent these issues, this study proposed a drone-based method for the rapid detection of deer. In addition, an experiment was conducted to detect the location of a deer wearing a collar node within a range of hundreds of meters. A drone can be used as a mobile base-station, to track the location of the fast moving deer based on vision recognition and signal tracking and to collect data accumulated in the collar node. The proposed AI detection method facilitated efficient data collection with less time required.

In addition, this study analyzed state-of-the-art AI pattern analysis algorithms, which were used to identify repeated patterns in the deer location data and interactions gleaned from the collar node. The analytical results of these algorithms can be used to identify repeated behaviors and patterns of animals more efficiently and accurately. In addition, this study examined various types of epidemic spread algorithms that have recently been developed. Both analytical results on pattern analysis and epidemic spread are expected to be combined and applied to prevent epidemics through animal behavior prediction. Our system and the intelligent researches were ultimately found to operate robustly in an outdoor wilderness environment for the duration of the research study. We believe that our design can be used to collect actual data for wild animal behavior tracking, if the base stations are installed in more locations and for longer period time, which increased the probability of data collection. We hope this Wildsense system can be used to assist wilderness biologists and ecologists in more precisely tracking and monitoring the movement and interaction patterns of free ranging deer and other wild animals in the future.

\section{References}

[1] J. Ahn, A. Mysore, K. Zybko, C. Krumm, S. Thokala, X. Xing, M. Lian, R. Han, S. Mishra, and T. Hobbs, "WildSense: Monitoring Interactions among Wild Deer in Harsh Outdoor Environments Using a Delay-Tolerant WSN," Journal of Sensors, vol. 2016, pp. 1-17, 2016. Article (CrossRef Link)

[2] R. J. Dsouza and J. Jose, "Routing approaches in delay tolerant networks: A survey," International Journal of Computer Applications, vol. 1, no. 17, pp. 8-14, 2010. Article (CrossRef Link)

[3] T. W. Grovenburg, J. A. Jenks, R. W. Klaver, C. C. Swanson, C. N. Jacques, and D. Todey, "Seasonal movements and home ranges of white-tailed deer in north central South Dakota," Canadian Journal of Zoology, vol. 89, no. 10, pp. 876-885, 2009. Article (CrossRef Link) 
[4] E. M. Schauber, C. K. Nielsen, L. J. Kjær, C. W. Anderson, and D. J. Storm, "Social affiliation and contact patterns among white-tailed deer in disparate landscapes: Implications for disease trans-mission," Journal of Mammal, vol. 96, no. 1, pp. 16-28, 2015. Article (CrossRef Link)

[5] D. L. Mech and M. S. Barber, "A Critique of Wildlife Radio-tracking and Its Use in National Parks," USGS Publications Warehouse; A Report to the U.S. National Park Service, 2002. Article (CrossRef Link)

[6] T. Wark, W. Hu, P. Sikka, L. Klingbeil, P. Corke, C. Crossmank, and G. Bishop-Hurley, "A model-based routing protocol for a mobile, delay tolerant network," in Proc. of the $5^{\text {th }}$ ACM International Conference on Embedded Networked Sensor Systems, pp. 421-422, 2007. Article (CrossRef Link)

[7] A. Pentland, R. Fletcher, and A. Hasson, "Daknet: Rethinking connectivity in developing nations," Computer, vol. 37, no. 1, pp. 78-83, 2004. Article (CrossRef Link)

[8] T. V. Prabhakar, A. Uttama, H. Jamadagni, K. Swaroop, R. V. Prasad, and I. M. Niemegeers, "A novel DTN based energy neutral transfer scheme for energy harvested WSN Gateways," ACM Sigmetrics Performance Evaluation Review, vol. 38, no. 3, pp. 71-75, 2011.

Article (CrossRef Link)

[9] M. Rutishauser, V. Petkov, T. M. Williams, C. Wilmers, J. Boice, K. Obraczka, and P. Mantey, "CARNIVORE: a disruption-tolerant system for studying wildlife," EURASIP Journal on Wireless Communications and Networking, 2011. Article (CrossRef Link)

[10] O. Iova, L. Murphy, G. P. Picco, L. Ghiro, D. Molteni, F. Ossi, and F. Cagnacci, "LoRa from the City to the Mountains: Exploration of Hardware and Environmental Factors," in Proc. of International Conference on Embedded Wireless Systems and Networks (EWSN '17), pp. 317-322, 2017. Article (CrossRef Link)

[11] T. Oliveira, M. Raju, and D. P. Agrawal, "Accurate Distance Estimation Using Fuzzy based combined RSSI/LQI Values in an Indoor Scenario: Experimental Verification," Network Protocols and Algorithms, vol. 4, no. 4, pp. 174-199, 2012. Article (CrossRef Link)

[12] G. Han, C. Zhang, J. Lloret, L. Shu, and J. P. C. J. Rodrigues, "A mobile anchor assisted localization algorithm based on regular hexagon in wireless sensor networks," The Scientific World Journal, vol. 2014, pp. 1-13, 2014. Article (CrossRef Link)

[13] R. Jurdak, P. Corke, D. Dharman, and G. Salagnac, "Adaptive gps duty cycling and radio ranging for energy-efficient localization," in Proc. of the $8^{\text {th }}$ ACM Conference on Embedded Networked Sensor Systems, pp. 57-70, 2010. Article (CrossRef Link)

[14] C. Liu, B. Li, D. Fang, S. Guo, X. Chen, and T. Xing, "Demo: Rhinopithecus roxellana monitoring and identification using wireless sensor networks," in Proc. of the $9^{\text {th }}$ ACM Conference on Embedded Networked Sensor Systems, pp. 427-428, 2011. Article (CrossRef Link)

[15] G. R. Karns, R. A. Lancia, C. S. DePerno, and M. C. Conner, "Investigation of adult male whitetailed deer excursions outside their home range," Southeastern Naturalist, vol. 10, no. 1, pp. 3952, 2011. Article (CrossRef Link)

[16] Crossbow MICAz. [Online]. Available: https://www.autex-open.com

[17] B. Kusy, P. Dutta, P. Levis, M. Maroti, A. Ledeczi, and D. Culler, "Elapsed time on arrival a simple and versatile primitive for canonical time synchronisation services," International Journal of Ad. Hoc Ubiquitous Computing, vol. 4, pp. 239-251, 2016. Article (CrossRef Link)

[18] J. Gwak, J. Jung, R. Oh, M. Park, M. A. K. Rakhimov, and J. Ahn, "A Review of Intelligent SelfDriving Vehicle Software Research," KSII Transactions on Internet and Information Systems, vol. 13, no. 11, pp. 5299-5320, 2019. Article (CrossRef Link)

[19] J. Jung, M. Park, K. Cho, C. Mun, and J. Ahn, "Intelligent Hybrid Fusion Algorithm with Vision Patterns for Generation of Precise Digital Road Maps in Self-driving Vehicles," KSII Transactions on Internet and Information Systems, vol. 14, no. 10, pp. 3955-3971, 2020. Article (CrossRef Link)

[20] S. Li, Z. Hu, and M. Zhao, "Moving Object Detection Using Sparse Approximation and Sparse Coding Migration," KSII Transactions on Internet and Information Systems, vol. 14, no. 5, pp. 2141-2155, 2020. Article (CrossRef Link) 
[21] P. Zhu, L. Wen, D. Du, X. Bian, H. Ling, Q. Hu, Q. Nie, H. Cheng, C. Liu, X. Liu, W. Ma, H. Wu, L. Wang, A. Schumann, and C. Brown, "VisDrone-DET2018: The Vision Meets Drone Object Detection in Image Challenge Results," in Proc. of European Conference on Computer Vision, pp. 437-468, 2018. Article (CrossRef Link)

[22] D. Dawei, P. Zhu, L. Wen, X. Bian, H. Lin, Q. Hu, T. Peng, J. Zheng, X. Wang, Y. Zhang, and L. Bo, "VisDrone-DET2019: The Vision Meets Drone Object Detection in Image Challenge Results," in Proc. of IEEE International Conference on Computer Vision Workshops, pp. 213-226, 2019. Article (CrossRef Link)

[23] T. Y. Lin, P. Dollar, R. Girshcik, K. He, B. Hariharan, and S. Belongie, "Feature Pyramid Networks for Object Detection," in Proc. of IEEE Conference on Computer Vision and Pattern Recognition, pp. 936-944, 2017. Article (CrossRef Link)

[24] T. Lin, P. Goyal, R. Girshick, K. He, and P. Dollar, "Focal loss for dense object detection," in Proc. of IEEE International Conference on Computer Vision, pp. 2999-3007, 2017. Article (CrossRef Link)

[25] N. Bodla, B. Singh, R. Chellappa, and L. S. Davis, "Soft-NMS--improving object detection with one line of code," in Proc. of IEEE International Conference on Computer Vision, pp. 5561-5569, 2017. Article (CrossRef Link)

[26] J. Hu, S. Albanie, G. Sun, and E. Wu, "Squeeze-and-excitation networks," Computer Vision and Pattern Recognition, 2017. Article (CrossRef Link)

[27] F. Wang, M. Jiang, C. Qian, S. Yang, C. Li, H. Zhang, X. Wang, and X. Tang, "Residual attention network for image classification," Computer Vision and Pattern Recognition, 2017. Article (CrossRef Link)

[28] S. Ren, K. He, R. Girshick, and J. Sun, "Faster r-cnn: Towards real-time object detection with region proposal networks," Computer Vision and Pattern Recognition, 2016. Article (CrossRef Link)

[29] Z. Cai and N. Vasconcelos, "Cascade r-cnn: Delving into high quality object detection," Computer Vision and Pattern Recognition, 2017. Article (CrossRef Link)

[30] K. He, G. Gkioxari, P. Dollar, and R. Girshcik, "Mask R-CNN," Computer Vision and Pattern Recognition, 2017. Article (CrossRef Link)

[31] B. Singh, M. Najibi, and L. S. Davis, "Sniper: Efficient multi-scale training," Computer Vision and Pattern Recognition, 2018. Article (CrossRef Link)

[32] S. Gidaris and N. Komodaks, "Object detection via a multi-region and semantic segmentationaware CNN model," Computer Vision and Pattern Recognition, 2015. Article (CrossRef Link)

[33] X. Zhou, D. Wang, and P. Krahenbuhl, "Objects as points," Computer Vision and Pattern Recognition, 2019. Article (CrossRef Link)

[34] Ynneec. [Online]. Available: https://www.yuneec.com/en_US/drones/typhoon-h/overview.html

[35] Walkera. [Online]. Available: https://www.walkera.com/index.php/Goods/info/id/24.html

[36] Skydio. [Online]. Available: https://www.skydio.com/pages/skydio-2

[37] Dji. [Online]. Available: https://www.dji.com/kr/mavic?site=brandsite\&from=landing page

[38] L. F. Hughey, A. M. Hein, A. Strandbur-Peshkin, and F. H. Jensen, "Challenges and solutions for studying collective animal behavior in the wild," Philosophical Transactions of the Royal Society B: Biological Sciences, vol. 373, no. 1746, 2018. Article (CrossRef Link)

[39] J. J. Valletta, C. Torney, M. Kings, A. Thornton, and J. Madden, “Applications of machine learning in animal behaviour studies,” Animal Behavior, vol. 124, pp. 203-220, 2017. Article (CrossRef Link)

[40] J. Rew, S. Park, Y. Cho, S. Jung, and E. Hwang, "Animal Movement Prediction Based on Predictive Recurrent Neural Network," Sensors, vol. 19, no. 20, 2019. Article (CrossRef Link)

[41] E. Browning, M. Bolon, E. Owen, A. Shoji, T. Guilford, and R. Freeman, "Predicting animal behaviour using deep learning: GPS data alone accurately predict diving in seabirds," Methods Ecollogy and Evolution, vol. 9, no. 3, pp. 681-692, 2018. Article (CrossRef Link)

[42] T. Michelot, R. Langrock, and T. A. Patterson, "moveHMM: an R package for the statistical modelling of animal movement data using hidden Markov models," Methods Ecollogy and Evolution, vol. 7, no. 11, pp. 1308-1315, 2016. Article (CrossRef Link) 
[43] S. Dabiri, C. Lu, K. Heaslip, and C. K. Reddy, "Semi-Supervised Deep Learning Approach for Transportation Mode Identification Using GPS Trajectory Data," IEEE Transactions on Knowledge and Data Engineering, vol. 32, no. 5, pp. 1010-1023, 2020. Article (CrossRef Link)

[44] F. Zong, Y. Tian, Y. He, J. Tang, and J. Lv, "Trip destination prediction based on multi-day GPS data," Physica A: Statistical Mechanics and Its Applications, vol. 515, pp. 258-269, 2019. Article (CrossRef Link)

[45] S. H. Kim, J. K. Choi, J. S. Kim, A. R. Jang, L. H. Lee, and K. J. Cha, "Animal Infectious Diseases Prevention through Big Data and Deep Learning," Journal of Intelligence and Information Systems, vol. 24, no. 4, pp. 137-154, 2018. Article (CrossRef Link)

[46] WHO. [Online]. Available: https://www.who.int/emergencies/diseases/novel-coronavirus-2019 ladvice-for-public

[47] M. M. Sajadi, P. Habibzadeh, A. Vintzileos, S. Shokouhi, F. Miralles-Wilhelm, and A. Amoros, "Temperature, Humidity and Latitude Analysis to Predict Potential Spread and Seasonality for COVID-19," SSRN, pp. 1-24, 2020. Article (CrossRef Link)

[48] M. Coccia, "Factors determining the diffusion of COVID-19 and suggested strategy to prevent future accelerated viral infectivity similar to COVID," Science of The Total Environment, vol. 729, 2020. Article (CrossRef Link)

[49] D. Balcan, H. Hu, B. Goncalves, P. Bajardi, C. Poletto, J. J. Ramasco, D. Paolotti, N. Perra, M. Tizzoni, W. V. D. Broeck, V. Colizza, and A. Vespignani, "Seasonal transmission potential and activity peaks of the new influenza $\mathrm{A}(\mathrm{H} 1 \mathrm{N1})$ : a Monte Carlo likelihood analysis based on human mobility," BMC Medicine, vol. 7, 2009. Article (CrossRef Link)

[50] P. Poletti, M. Ajelli, and S. Merler, "The Effect of Risk Perception on the 2009 H1N1 Pandemic Influenza Dynamics," PLOS ONE, vol. 6, no. 2, 2011. Article (CrossRef Link)

[51] M. U. G. Kraemer, N. Golding, D. Bisanzio, S. Bhatt, D. M. Pigott, S. E. Ray, O. J. Brady, J. S. Brownstein, N. R. Faria, D. A. T. Cummings, O. G. Pybus, D. L. Smith, A. J. Tatem, S. I. Hay, and R. C. Reiner, "Utilizing general human movement models to predict the spread of emerging infectious diseases in resource poor settings," Scientific Report, vol. 9, 2019. Article (CrossRef Link)

[52] Z. Yang, Z. Zeng, K. Wang, S. Wong, W. Liang, M. Zanin, P. Liu, X. Cao, Z. Gao, Z. Mai, J. Liang, X. Liu, S. Li, Y. Li, F. Ye, W. Guan, Y. Yang, F. Li, S. Luo, Y. Xie, B. Liu, Z. Wang, S. Zhang, Y. Wang, N. Zhong, and J. He, "Modified SEIR and AI prediction of the epidemics trend of COVID-19 in China under public health interventions," Journal of Thoracic Disease, vol. 12, no. 3, pp. 165-174, 2020. Article (CrossRef Link)

[53] I. Cooper, A. Mondal, and C. G. Antonopoulos, "A SIR model assumption for the spread of COVID-19 in different communities," Chaos, Solitons \& Fractals, vol. 139, 2020. Article (CrossRef Link)

[54] J. Wangping, H. Ke, Y. Song, W. Cao, S. Wang, S. Yang, J. Wang, F. Kou, P. Tai, J. Li, M. Liu, and Y. He, "Extended SIR Prediction of the Epidemics Trend of COVID-19 in Italy and Compared With Hunan," Frontiers in Medicine, vol. 7, 2020. Article (CrossRef Link)

[55] Drone Life. [Online]. Available: https://dronelife.com/2020/08/01/a-drone-battery-that-chargesin-5-minutes/ 

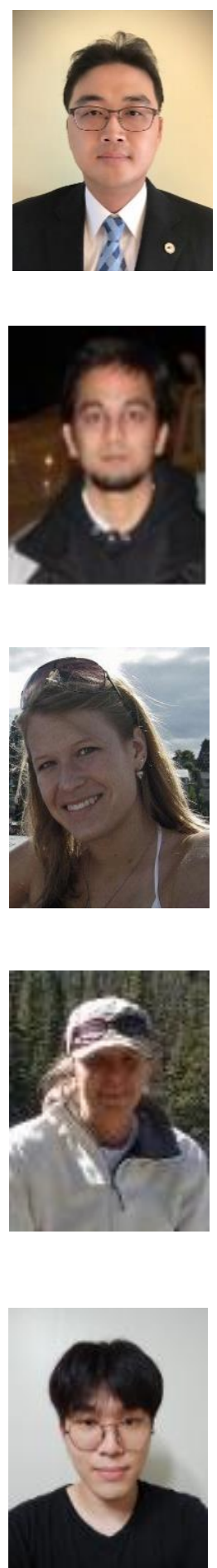

Jun-Ho Ahn is an Associate Professor in the Computer Information Technology at Korea National University of Transportation. Junho Ahn received a Ph.D. degree in Computer Science at University of Colorado at Boulder in 2013. Junho Ahn is interested in intelligent extensive knowledge of vision, artificial intelligence algorithms, self-driving car systems, mobile systems, embedded systems, sensor networks, and the prospects for uniting these areas. Much of his research involved intelligent mobile and self-driving car application systems, in which he designed to intelligent fuse multi-modal mobile sensor data.

Akshay Mysore received the B.S. and M.S. degrees in Electronics and Communication Engineering and Electrical Engineering from National Institute of Technology Durgapur and University of Colorado at Boulder, USA in 2011 and 2014, respectively.

Kati Zybko received the B.S. in the Department of Natural Resources at Colorado State University. she was majoring in Conservation Biology and got her minor in Spanish. She worked as a field technician for the project. She is working in Wildlands Studies.

Caroline Krumm received her Bachelor's in Wildlife Biology and a Master's in the Graduate Degree Program in Ecology from Colorado State University. She has worked on various studies of chronic wasting disease in deer since 1998, including a study examining selective predation by mountain lions on diseased prey. Her interests are disease and predator-prey dynamics in wildlife populations worldwide.

Do-Hyeon Lee is an integrated B.S./M.S. student in the Department of Software at Korea Transportation University. $\mathrm{He}$ is interested in intelligence algorithms and Pattern Recognition. 


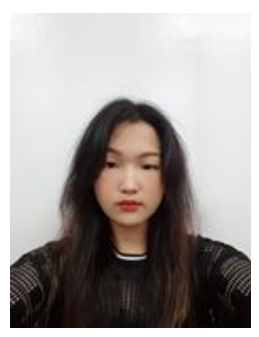

Da-Hyeon Kim is an integrated B.S./M.S. student in the Department of Software at Korea Transportation University. She is interested in Computer Vision, Deep Learning, and Autonomous Driving.

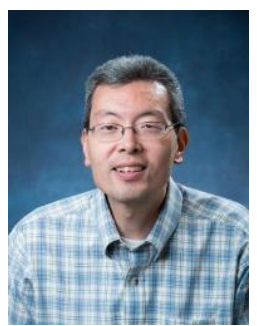

Richard Han is a Professor in University of Colorado at Boulder Department of Computer Science. He received a Ph.D. degree in Electrical Engineering at University of California at Berkeley, USA in 1997. He received the B.S. and M.S. degrees in Electrical Engineering and Electrical Engineering from Stanford University and University of California at Berkeleyin 1989 and 1991, respectively.

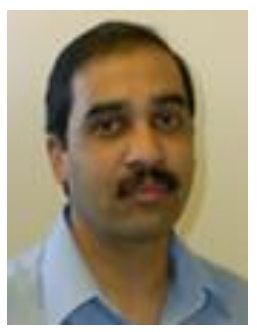

Shivakant Mishra is a Professor in University of Colorado at Boulder Department of Computer Science. He received a Ph.D. degree in Computer Science, University of Arizona, USA.

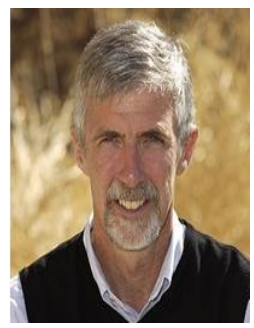

Thompson Hobbs is a Professor in Natural Resource Ecology Laboratory at Colorado State University. He received a Ph.D. degree in Wildlife Biology at Colorado State University, USA in 1979. He received the B.A. and M.S. degrees in Biology and Wildlife Biology from Grinnell College and Colorado State University in 1974 and 1977, respectively. 